Pacific Journal of Mathematics

NORMED FIELDS WHICH EXTEND NORMED RINGS O 


\title{
NORMED FIELDS WHICH EXTEND NORMED RINGS OF INTEGERS
}

\author{
Silvio AURORA
}

\begin{abstract}
It is shown that if the ring of integers is made a normed ring by using a "reasonable" norm, such as the ordinary absolute value or some power thereof, then every normed field which extends such a normed ring is a subfield of the field of complex numbers.
\end{abstract}

The development of the foundations of analysis involves the construction of the normed field of complex numbers, with the ordinary absolute value as norm, from the normed ring of integers, with the ordinary absolute value as norm, by a process of successive enlargements of algebraic systems. (By a normed ring is meant a ring $A$ which is provided with a norm function $N$; that is, $N$ is a real-valued function defined on $A$ such that: (i) $N(0)=0$ and $N(x)>0$ for every nonzero $x$ in $A$, (ii) $N(-x)=N(x)$ for all $x$ in $A$, (iii) $N(x+y) \leqq$ $N(x)+N(y)$ for all $x, y$ in $A$, (iv) $N(x y) \leqq N(x) N(y)$ for all $x, y$ in $A$.) Although some treatments of this construction create only positive numbers in the early stages of the passage from the system of natural numbers to the complex number system, such approaches could easily be modified to retain their basic features while still producing the ring of integers at the outset; thus, all such procedures essentially involve the extension of the normed ring of integers to produce the normed field of complex numbers.

One might ask what normed fields could be produced by enlarging the normed ring of integers, with the ordinary absolute value or some power thereof as norm, if no restriction whatever were placed upon the method of extension. It is shown in Theorem 3 that the only normed fields which can be thus obtained must be (continuously isomorphic to) subfields of the field of complex numbers.

Somewhat similar results are given in $\S 4$ for the situation in which the normed field of rational numbers, with a suitably "natural" norm, is enlarged to create a new normed field. For instance, the corollary of Theorem 6 indicates that if the field of rational numbers is provided with a norm which coincides with a power of the ordinary absolute value over a suitable neighborhood of zero, then every normed field which extends this normed field is (continuously isomorphic to) a subfield of the field of complex numbers.

2. Preliminaries. It is useful to recall some of the concepts which are employed in [1] and [2]. 
A norm for a ring $A$ is a real-valued function $N$ defined on $A$ such that: (i) $N(0)=0$ and $N(x)>0$ for all nonzero $x$ in $A$, (ii) $N(-x)=N(x)$ for all $x$ in $A$, (iii) $N(x+y) \leqq N(x)+N(y)$ for all $x, y$ in $A$, (iv) $N(x y) \leqq N(x) N(y)$ for all $x, y$ in $A$. If a norm $N$ for a ring $A$ has the property that $N(x y)=N(x) N(y)$ for all $x, y$ in $A$ then $N$ is called an absolute value for $A$.

By a normed ring is meant a ring $A$, together with a norm $N$ for $A$; the norm for a normed ring $A$ defines a metric, and therefore a topology, for $A$.

If $N$ is a norm for a ring $A$ and $c$ is an element of $A$ such that $N(c x)=N(c) N(x)$ for all $x$ in $A$ then $N$ is said to be homogeneous at c. $A$ norm $N$ for a ring $A$ is said to be power multiplicative at an element $c$ of $A$ provided that $N\left(c^{n}\right)=N(c)^{n}$ for every natural number $n$. When a norm $N$ for a ring $A$ is homogeneous (power multiplicative) at every element of a subset $C$ of $A$ then $N$ is said to be homogeneous (power multiplicative) on $C$.

In case $N$ and $N^{\prime}$ are norms for a ring $A$ such that $N^{\prime}(x) \leqq N(x)$ for all $x$ in $A$ then we shall write $N^{\prime} \leqq N$. The relation $\leqq$ in the set of norms for a ring $A$ constitutes a partial ordering of that set.

An example will serve to illustrate some of these concepts. Let $A$ be the ring of all real functions which are defined and have a continuous derivative on the closed unit interval [0,1]. If $N^{\prime}(x)=$ $\sup \{|x(t)| \mid 0 \leqq t \leqq 1\}$ and

$$
N(x)=\sup \{|x(t)| \mid 0 \leqq t \leqq 1\}+\sup \left\{\left|x^{\prime}(t)\right| \mid 0 \leqq t \leqq 1\right\}
$$

for all $x$ in $A$, then $N^{\prime}$ and $N$ are norms for $A$, with $N^{\prime} \leqq N$. It is also easily established that $N^{\prime}$ is power multiplicative on $A$ and that $N$ is homogeneous at each constant function which belongs to $A$.

When $N$ is a norm for a field $K$ and $c$ is a nonzero element of $K$, then for all $x$ in $K$ :

$$
N(x) \geqq N(x c) / N(c) \geqq N\left(x c^{2}\right) / N(c)^{2} \geqq N\left(x c^{3}\right) / N(c)^{3} \geqq \cdots
$$

Thus

$$
N_{c}(x)=\inf \left\{N\left(x c^{n}\right) / N(c)^{n} \mid n \text { a natural number }\right\}=\lim _{n \rightarrow \infty} N\left(x c^{n}\right) / N(c)^{n}
$$

is a well-defined nonnegative real number for all $x$ in $A$. It can be shown that the function $N_{c}$ is identically zero on $A$ if and only if $N$ fails to be power multiplicative at $c$. On the other hand, if $N$ is power multiplicative at $c$ then $N_{c}$ is a norm for $K$, with $N_{c} \leqq N$, as the following lemma indicates. (It is recalled that by a semigroup in a ring is meant a nonempty subset of that ring such that the subset is closed under multiplication.) 
Lemma 1. Let $N$ be a norm for a field $K$, and let $c$ be a nonzero element of $K$ such that $N$ is power multiplicative at c. Then $N_{c}$ is a norm for $K$ such that:

(i) $N_{c} \leqq N$,

(ii) $N_{c}(c)=N(c)$,

(iii) $N_{c}$ is homogeneous at $c$,

(iv) whenever $S$ is a semigroup in $K$, with $c$ in $S$, such that $N$ is power multiplicative on $S$ then $N_{c}$ is power multiplicative on $S$.

It is easily established that $N_{c}$ possesses properties (ii), (iii), (iv) of a norm, so that the set $I$ of all $x$ in $A$ for which $N_{c}(x)=0$ is an ideal in the field $K$, and $N_{c}$ is therefore a norm for $K$. The remaining details of the proof are routine.

The lemma permits us to replace the norm $N$ by a new norm which has properties similar to those of $N$ and is homogeneous at $c$ as well. It is possible to sharpen this result so that the new norm is homogeneous on an entire semigroup on which the original norm is power multiplicative.

THEOREM 1. Let $K$ be a normed field with norm $N$, let $S$ be a semigroup in $K$ such that $N$ is power multiplicative on $S$, and let $c$ be a nonzero element of $S$. Then there exists a norm $N^{\prime}$ for $K$ such that:

(i ) $N^{\prime} \leqq N$,

(ii) $N^{\prime}(c)=N(c)$,

(iii) $N^{\prime}$ is homogeneous on $S$.

Proof. Let $\mathscr{H}$ be the set of all norms $N^{\prime \prime}$ for $K$ such that $N^{\prime \prime} \leqq N, N^{\prime \prime}(c)=N(c), N^{\prime \prime}$ is homogeneous at $c$, and $N^{\prime \prime}$ is power multiplicative on $S$. Then $\mathscr{H}$ is not empty since it contains $N_{c}$; also, $\mathscr{C}$ is partially ordered by the relation $\leqq$. It is easily shown that every totally ordered subset of $\mathscr{C}$ has a lower bound in $\mathscr{H}$, so that Zorn's Lemma implies the existence of a minimal element, $N^{\prime}$, of $\mathscr{H}$.

If $d$ is a nonzero element of $S$ then Lemma 1 implies that $\left(N^{\prime}\right)_{d}$ belongs to $\mathscr{H}$, with $\left(N^{\prime}\right)_{d}(d x)=\left(N^{\prime}\right)_{d}(d) \cdot\left(N^{\prime}\right)_{d}(x)$ for all $x$ in $K$. Since $N^{\prime}$ is a minimal element of $\mathscr{H}$, and since $N^{\prime}$ and $\left(N^{\prime}\right)_{d}$ both belong to $\mathscr{H}$, with $\left(N^{\prime}\right)_{d} \leqq N^{\prime}$, it follows that $N^{\prime}=\left(N^{\prime}\right)_{d}$. Thus, $N^{\prime}(d x)=$ $N^{\prime}(d) N^{\prime}(x)$ for all $x$ in $K$. We conclude that $N^{\prime}$ is homogeneous at every element $d$ of $S$, and the theorem follows.

REMARK. In order to apply Theorem 1 it is useful to have a criterion to determine when a norm for a ring is power multiplicative on a semigroup in that ring. It is easily established that a norm $N$ 
for a ring $A$ is power multiplicative on a semigroup $S$ in $A$ if and only if for every element $x$ in $S$ there is an integer $n(x)$, with $n(x)>1$, such that $N\left(x^{n(x)}\right)=N(x)^{n(x)}$. In particular, $N$ is power multiplicative on $S$ if and only if $N\left(x^{2}\right)=N(x)^{2}$ for all $x$ in $S$. (Any integer exponent greater than 1 could be used instead of 2 in the preceding statement.)

3. Extensions of the normed ring of integers. We are interested in normed fields which extend the ring of integers when the latter is provided with a norm which is a power of the ordinary absolute value. It will be shown that such fields are (continuously isomorphic to) subfields of the field of complex numbers. First a more general result is obtained which implies that if the ring of integers is given a norm which is power multiplicative and takes a value greater than 1 at least once then any normed field which extends this normed ring must be (continuously isomorphic to) a subfield of the field of complex numbers.

For convenience, whenever $n$ is an integer the symbol $n$ will be used to denote the $n$-fold of the unit element of the field which is under consideration.

THEOREM 2. Let $K$ be a normed field for which there is a natural number $n_{0}$, with $N\left(n_{0}\right)>1$, such that $N\left(n^{2}\right)=N(n)^{2}$ whenever $n$ is a natural number for which $n \geqq n_{0}$. Then $K$ is continuously algebraically isomorphic to a subfield of the field (5) of complex numbers.

Proof. If $S$ is the set of all elements $n$ of $K$ such that $n$ is a natural number with $n \geqq n_{0}$, then $S$ is a semigroup in $K$ such that $N$ is power multiplicative on $S$. Theorem 1 can be applied to the semigroup $S$ and the element $n_{0}$ in order to obtain a norm $N^{\prime}$ for $K$ such that $N^{\prime} \leqq N, N^{\prime}\left(n_{0}\right)=N\left(x_{0}\right)>1$, and $N^{\prime}$ is homogeneous on $S$.

If $n$ is an arbitrary natural number greater than 1 then there is a natural number $r$ such that $n^{r}$ and $n^{r+1}$ both belong to $S$; the inequality $\quad N^{\prime}\left(n^{r}\right) N^{\prime}(n) N^{\prime}(x)=N^{\prime}\left(n^{r+1}\right) N^{\prime}(x)=N^{\prime}\left(n^{r+1} x\right) \leqq N^{\prime}\left(n^{r}\right) N^{\prime}(n x)$ implies that $N^{\prime}(n x)=N^{\prime}(n) N^{\prime}(x)$ for all $x$ in $K$. From the condition $N^{\prime}\left(n_{0} x\right)=N^{\prime}\left(n_{0}\right) N^{\prime}(x)$ with $x=1$ we obtain $N^{\prime}(1)=1$, and consequently $N^{\prime}$ is homogeneous at every "integer" in $K$. Thus $N^{\prime}$ is homogeneous on the prime field, $P$, of $K$. Since $N^{\prime}\left(n_{0}\right)>1$, the restriction of $N^{\prime}$ to $P$ is an archimedean absolute value for $P$; therefore Ostrowski's results [4] imply that $P$ is algebraically isomorphic to the field of rationals (and can be identified with that field), and there is a real number $s$, with $0<s \leqq 1$, such that $N^{\prime}(x)=|x|^{s}$ for all $x$ in $P$.

Let $A$ be the completion of $K$ relative to the norm $N^{\prime}$, so that 
$A$ is a complete commutative normed ring with unity, and there is an obvious continuous isomorphism $\varphi$ of $K$ into $A$. We have in fact $N^{\prime \prime}(\varphi(x))=N^{\prime}(x) \leqq N(x)$ for all $x$ in $K$ if $N^{\prime \prime}$ is the norm for $A$. The closure, $R$, of $\varphi(P)$ in $A$ is the completion of $\varphi(P)$ and can be identified with the completion of $P$. Therefore $R$ can be identified with the field of real numbers, and we have $N^{\prime \prime}(y)=|y|^{s}$ for all $y$ in $R$.

There is a maximal ideal $M$ in $A$, and $M$ is closed since the set of invertible elements of a complete normed ring with unity is open. Thus, $A / M$ is a complete normed field and has its norm $\bar{N}$ given by the rule $\bar{N}(X)=\inf \left\{N^{\prime \prime}(x) \mid x \in X\right\}$ for all $X$ in $A / M$. The natural homomorphism $\nu$ of $A$ onto $A / M$ is continuous since $\bar{N}(\nu(y)) \leqq N^{\prime \prime}(y)$ for all $y$ in $A$, and $\nu(R)$ is therefore identifiable with the field $R$. Then $A / M$ may be considered a complete commutative normed division algebra over $R$, where $R$ is the field of real numbers with a power of the ordinary absolute value as its absolute value. The GelfandMazur Theorem, as it appears in [3; Chap. 6, p. 127, Th. 1], implies that $A / M$ is continuously isomorphic to the field of real numbers or the field of complex numbers, so that there is a continuous isomorphism $\psi$ of $A / M$ into the field 5 of complex numbers.

It is easily seen that the mapping $\psi \circ \nu \circ \varphi$ is a continuous isomorphism of the field $K$ into $\mathfrak{C}$, and the theorem follows.

Note. An alternative means of stating Theorem 2 is that if the ring of integers is given a norm which is power multiplicative at every integer which is sufficiently large, and if the norm takes a value greater than 1 for at least one of those integers, then every normed field which is an extension of this normed ring must be a subfield of 5 with a topology at least as fine as its ordinary relative topology in $\sqrt{5}$.

The simplest norms which satisfy the hypothesis of Theorem 2 are those which coincide with some power of the ordinary absolute value at all natural numbers which are sufficiently large. We thus obtain the following theorem.

THeOREM 3. Let $K$ be a normed field for which there exist a natural number $n_{0}$ and a positive real number $s$ such that $N(n)=n^{s}$ whenever $n$ is a natural number with $n \geqq n_{0}$. Then $K$ is continuously algebraically isomorphic to a subfield of $\mathfrak{}$.

It should be noted that $s$ is necessarily less than or equal to 1 . A special case of Theorem 3, that in which $s=1$, has been given in [2; Corollary 2 of Th. 5]. Another result of some interest can be obtained as a corollary of the theorem, and has appeared in [2; Th. 6]. 
COROLLARY. Let $K$ be a normed field such that $N(n)=n N(1)$ for infinitely many natural numbers $n$. Then $K$ is continuously algebraically isomorphic to a subfield of $\mathfrak{c}$.

The proof involves replacement of the norm $N$ by a new norm $N^{\prime}$ defined by $N^{\prime}(x)=\sup \{N(x c) / N(c) \mid c \in K, c \neq 0\}$ for all $x$ in $K$.

Note. Theorem 3 implies that if the ring of integers is provided with a norm which is a power of the ordinary absolute value (or if the norm merely coincides with some power of the ordinary absolute value at integers which are sufficiently large) then every normed field which extends this normed ring must be a subfield of $\mathbb{5}$ with a topology at least as fine as its ordinary relative topology.

An interesting consequence of these results concerns normed fields which satisfy the parallelogram law.

Definition. A normed ring $A$ is said to satisfy the parallelogram $l a w$ if $N(x+y)^{2}+N(x-y)^{2}=2 N(x)^{2}+2 N(y)^{2}$ whenever $x, y$ belong to $A$.

The parallelogram law is characteristic of Euclidean distance and can hold for a normed field only if that field is continuously embeddable in the field of complex numbers.

THEOREM 4. Let $K$ be a normed field which satisfies the parallelogram law. Then $K$ is continuously algebraically isomorphic to a subfield of $\mathfrak{c}$.

Proof. The parallelogram law with $x=y$ yields the relation $N(2 x)=2 N(x)$ for all $x$ in $K$. Thus, $N\left(2^{r} x\right)=2^{r} N(x)$ for all $x$ in $K$ and for every natural number $r$. The corollary of the preceding theorem then leads to the desired result.

4. Extensions of the normed field of rational numbers. The fields of the preceding section were all necessarily of infinite characteristic although the hypotheses employed in the statements of the results did not explicitly make such an assumption. We now confine our attention to fields of infinite characteristic, and the discussion is simplified by identifying the prime field of each such field with the field of rational numbers. The results of this section then indicate that if the field of rational numbers is given a norm which is "reasonable" in an appropriate sense, then every normed field which extends 
such a normed field must be (continuously isomorphic to) a subfield of (5.

We first obtain an analogue of Theorem 2.

THEOREM 5. Let $K$ be a normed field of infinite characteristic for which there is a natural number $n_{0}$, with $N\left(1 / n_{0}\right)<1$, such that $N\left(1 / n^{2}\right)=N(1 / n)^{2}$ whenever $n$ is a natural number with $n \geqq n_{0}$. Then $K$ is continuously algebraically isomorphic to a subfield of 5 .

Proof. If $S$ is the semigroup which consists of the elements $1 / n$ of $K$ for which $n$ is a natural number with $n \geqq n_{0}$, then $N$ is power multiplicative on $S$ and we may apply Theorem 1 to $S$ and the element $1 / n_{0}$. Thus, there is a norm $N^{\prime}$ for $K$, with $N^{\prime} \leqq N$, such that $N^{\prime}$ is homogeneous on $S$ and $N^{\prime}\left(1 / n_{0}\right)=N\left(1 / n_{0}\right)<1$. We have $N^{\prime}\left(n_{0}\right)>1$ since $N^{\prime}\left(1 / n_{0}\right)<1$. Also, whenever, $n$ is a natural number with $n \geqq n_{0}$ then $N^{\prime}\left(1 / n^{2}\right) N^{\prime}\left(n^{2}\right)=1=N^{\prime}(1 / n)^{2} N^{\prime}(n)^{2}=N^{\prime}\left(1 / n^{2}\right) N^{\prime}(n)^{2}$, so that $N^{\prime}\left(n^{2}\right)=N^{\prime}(n)^{2}$. Thus, $K$ with the norm $N^{\prime}$ satisfies the hypothesis of Theorem 2, and the theorem follows since $K$ is continuously algebraically isomorphic to this normed field.

When the norm for a normed field of infinite characteristic coincides with some power of the ordinary absolute value at the reciprocals of all natural numbers which are sufficiently large, we obtain an analogue of Theorem 3 .

THeOREm 6. Let $K$ be a normed field of infinite characteristic for which there exist a natural number $n_{0}$ and a positive real number $s$ such that $N(1 / n)=1 / n^{s}$ whenever $n$ is a natural number with $n \geqq n_{0}$. Then $K$ is continuously algebraically isomorphic to a subfield of $\mathfrak{c}$.

COROLlaRY. Let $K$ be a normed field of infinite characteristic for which there exist positive real numbers $r_{0}$ and $s$ such that $N(r)=r^{\text {s }}$ whenever $r$ is a rational number with $0<r<r_{0}$. Then $K$ is continuously algebraically isomorphic to a subfield of 5 .

We note that the corollary implies that if the field of rational numbers is provided with a norm which coincides with some power of the ordinary absolute value over a suitable neighborhood of zero, then every normed field which can be obtained by extending this normed field must be a subfield of $\sqrt{5}$ with a topology at least as fine as its ordinary relative topology in $\mathfrak{C}$. The special case of this corollary which occurs when $s=1$ has already been given in [2; Th. 7]. 
REMARK. Theorems 2, 3, 5, and 6 and their corollaries identify the normed field $K$ with a subfield of the field 5 of complex numbers, but with a topology finer than the ordinary topology inherited from 5. That the topology for $K$ may be strictly finer than the ordinary topology is shown by taking as $K$ the field of complex numbers with the norm $N$ given by $N(x)=\max (|x|,|\sigma(x)|)$ for every complex number $x$, where $\sigma$ is a fixed discontinuous automorphism of the field of complex numbers.

\section{REFERENCES}

1. S. Aurora, Multiplicative norms for metric rings, Pacific J. Math. 7 (1957), 12791304.

2. — The embedding of certain metric fields, Michigan Math. J. 7 (1960), 123128.

3. N. Bourbaki, "Algèbre commutative," chap. 5-6, Éléments de mathématique, Hermann, Paris, 1964.

4. A. Ostrowski, Ueber einige Loesungen der Funktionalgleichung $\phi(x) \cdot \phi(y)=\phi(x y)$. Acta Math. 41 (1918), 271-284.

Received April 9, 1969.

RUTGERS UNIVERSITY

NeWARK, NEW JERSEY 


\section{PACIFIC JOURNAL OF MATHEMATICS}

\section{EDITORS}

H. SAMELSON

Stanford University

Stanford, California 94305

\section{RichaRd PIERCe}

University of Washington

Seattle, Washington 98105
J. DUGUNDJI

Department of Mathematics

University of Southern California

Los Angeles, California 90007

RICHARD ARENS

University of California

Los Angeles, California 90024

\section{ASSOCIATE EDITORS}

E. F. BECKENBACH

B. H. NeUManN

F. WOLF

K. YosHIDA

\section{SUPPORTING INSTITUTIONS}

UNIVERSITY OF BRITISH COLUMBIA CALIFORNIA INSTITUTE OF TECHNOLOGY UNIVERSITY OF CALIFORNIA MONTANA STATE UNIVERSITY

UNIVERSITY OF NEVADA

NEW MEXICO STATE UNIVERSITY

OREGON STATE UNIVERSITY

UNIVERSITY OF OREGON

OSAKA UNIVERSITY

UNIVERSITY OF SOUTHERN CALIFORNIA
STANFORD UNIVERSITY

UNIVERSITY OF TOKYO

UNIVERSITY OF UTAH

WASHINGTON STATE UNIVERSITY

UNIVERSITY OF WASHINGTON

${ }^{*} \quad{ }^{*} \quad{ }^{*}$
AMERICAN MATHEMATICAL SOCIETY
CHEVRON RESEARCH CORPORATION
TRW SYSTEMS
NAVAL WEAPONS CENTER




\section{Pacific Journal of Mathematics}

\section{Vol. 33, No. $1 \quad$ March, 1970}

Mir Maswood Ali, On some extremal simplexes ................... 1

Silvio Aurora, On normed rings with monotone multiplication........... 15

Silvio Aurora, Normed fields which extend normed rings of integers....... 21

John Kelly Beem, Indefinite Minkowski spaces..................... 29

T. F. Bridgland, Trajectory integrals of set valued functions ........... 43

Robert Jay Buck, A generalized Hausdorff dimension for functions and sets ......................................... 69

Vlastimil B. Dlab, A characterization of perfect rings . . . . . . . . . . . . 79

Edward Richard Fadell, Some examples in fixed point theory ............ 89

Michael Benton Freeman, Tangential Cauchy-Riemann equations and uniform approximation ............................. 101

Barry J. Gardner, Torsion classes and pure subgroups ................ 109

Vinod B. Goyal, Bounds for the solution of a certain class of nonlinear

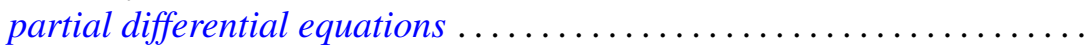

Fu Cheng Hsiang, On C, 1 summability factors of Fourier series at a given

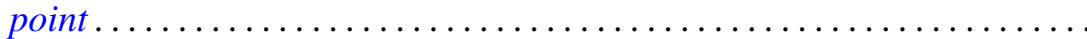

Lawrence Stanislaus Husch, Jr., Homotopy groups of PL-embedding

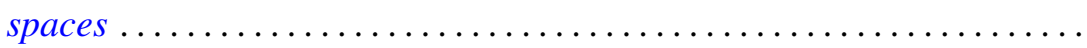

Daniel Ralph Lewis, Integration with respect to vector measures..........

Marion-Josephine Lim, $\mathscr{L}-2$ subspaces of Grassmann product spaces

Stephen J. Pierce, Orthogonal groups of positive definite multilinear functionals

W. J. Pugh and S. M. Shah, On the growth of entire functions of bounded index.

Siddani Bhaskara Rao and Ayyagari Ramachandra Rao, Existence of triconnected graphs with prescribed degrees . . .

Ralph Tyrrell Rockafellar, On the maximal monotonicity of subdifferential

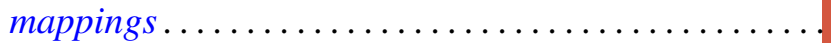

R. Shantaram, Convergence of a sequence of transformations of distribution functions. II ...............................

Julianne Souchek, Rings of analytic functions..............

Ted Joe Suffridge, The principle of subordination applied to functions of several variables...

Wei-lung Ting, On secondary characteristic classes in cobordism

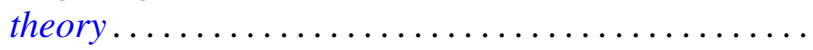

Pak-Ken Wong, Continuous complementors on $B^{*}$-algebras ...

Miyuki Yamada, On a regular semigroup in which the idempotents form a band. 